\title{
In-vivo Molecular Investigations of Live Tissues Using Diffracting Sources
}

\author{
Vasilis Ntziachristos, Jorge Ripoll, Edward Graves, and Ralph Weissleder \\ Center for Molecular Imaging Research \\ Massachusetts General Hospital and Harvard Medical School Boston MA \\ \{vasilis, ripoll, graves, weissleder\}@helix.mgh.harvard.edu
}

\begin{abstract}
We present novel technologies based on reconstructions employing diffracting sources in fluorescent mode that resolve molecular function in deep tissues. To facilitate time-efficient three-dimensional reconstructions we have employed fast analytical solutions of the diffusion equation to allow for close to real-time volumetric imaging of fluorescent near-infrared beacons that activate (fluoresce) with high specificity in the presence of specific molecular targets.
\end{abstract}

\section{Introduction}

Systematic efforts are under way to develop fluorescent markers and imaging systems that would allow in-vivo examinations of pathogenesis and of treatment at the molecular level [1]. The rationale for developing such technologies is a) to elucidate molecular mechanisms of disease in unperturbed environments over time, b) to allow earlier detection of disease based on molecular targets and c) to permit design of patient-specific treatments and monitor therapies on their molecular effect. Fluorescence imaging has recently seen significant growth due to its ability in assessing protein function and gene expression in-vivo [2]. Several elegant technologies that evolved to assess the fluorescence emitted from extrinsically administered fluorescent probes or markers or the expression of fluorescent proteins from engineered cells. Traditionally, fluorescence investigations have been performed by microscopic observations of surface or subsurface $(0-500 \mu \mathrm{m})$ fluorescence using confocal microscopy and multi-photon microscopy. Both techniques have allowed unprecedented insights in in-vivo imaging biology at the cellular or subcellular level $[3,4]$. A similar technology, evanescent wave microscopy, has also been used for fluorescent investigations of cell membranes [5]. However for clinical implementations it would be advantageous to develop technologies that could probe deeper in tissue and elucidate molecular function of tissues in-vivo based on highly specific fluorescent molecular probes.

Herein we present the implementation of three-dimensional fluorescence-mediated molecular tomography (FMT), a novel technique that is based on the general principles of tomography with diffracting sources $[6,7]$ and can probe molecular function deep in tissues $(0.2-12 \mathrm{~cm})$. The technique shines light through tissue at different projections and concurrently utilizes intrinsic and fluorescence 
measurements to provide quantitative images of fluorescent molecular probes. High molecular specificity and deep tissue penetrations can be achieved by capitalizing on activatable probes [8, 9] (molecular beacons) i.e. quenched (dark) probes that fluoresce only upon interaction with certain molecular targets offering great background signal suppression. Of particular importance are recent advances in quantitatively solving the inversion problem without need of additional measurements besides in-vivo measurements from the tissue of interest in fluorescent and intrinsic mode and the reduction of the computational expense in calculating complex boundaries. These two developments have facilitated the implementation of FMT systems in three-dimensional imaging schemes of murine models and could propagate in human applications as well.

In the following we discuss the algorithmic advances that allow the implementation of time-efficient forward problems and subsequent inversion and demonstrate the capacity of the technology to resolve protease activity in-vivo attaining inversion times of less than 3 minutes for the three-dimensional animal imaging problem.

\section{Theory}

Fluorescence-mediated tomography is based on a self-calibrated algorithm developed specifically for imaging fluorochrome distribution in-vivo [10]. The algorithm has been implemented into functional code using the Matlab software (Mathworks Inc) and combines a normalized-Born algorithm with the tangent-plane method, which is an analytical time-efficient approximation for solving the forward problem in the presence of complex boundaries. The algorithmic specifics are presented in the following:

\subsection{Normalized-Born Expansion}

In its general form, the forward problem can be written as an integral equation that related the unknown fluorochrome distribution $n(\vec{r})$ to a synthetic measurement $U_{s}\left(\vec{r}_{s}, \vec{r}_{d}\right)$ detected at position $\vec{r}_{d}$ due to a source at position $\vec{r}_{s}$, which is written as

$$
\begin{aligned}
& U_{s}\left(\vec{r}_{s}, \vec{r}_{d}\right)=S_{0} \cdot \frac{U_{f l}\left(\vec{r}_{s}, \vec{r}_{d}\right)-U_{b l}\left(\vec{r}_{s}, \vec{r}_{d}\right)}{U_{i n c}\left(\vec{r}_{s}, \vec{r}_{d}\right)}= \\
& =\frac{1}{U_{0}\left(\vec{r}_{s}, \vec{r}_{d}, k^{\lambda 1}\right)} \int d^{3} r \cdot\left(U_{0}\left(\vec{r}_{s}-\vec{r}, k^{\lambda 1}\right) \cdot n(\vec{r}) \cdot \frac{v}{D^{\lambda 2}} G\left(\vec{r}_{d}-\vec{r}, k^{\lambda 2}\right)\right.
\end{aligned}
$$

where $U_{f l}\left(\vec{r}_{s}, \vec{r}_{d}\right), U_{i n c}\left(\vec{r}_{s}, \vec{r}_{d}\right)$ are measurements at the emission and excitation wavelength respectively, $U_{b l}\left(\vec{r}_{s}, \vec{r}_{d}\right)=\Theta_{f} \cdot U_{i n c}\left(\vec{r}_{s}, \vec{r}_{d}\right)$ is the bleed-through signal (i.e. intrinsic signal not perfectly filtered out by the fluorescence band-pass filters), 
$\Theta_{f}$ is the band-pass filter attenuation factor, $S_{0}$ is a gain term that accounts for instrument gain differences at the excitation $\left(\lambda_{1}\right)$ and emission $\left(\lambda_{2}\right)$ wavelengths, $n(\vec{r})$, the fluorochrome distribution, is the product of the fluorochrome absorption coefficient and fluorescence quantum yield, $k^{\lambda 1}, k^{\lambda 2}$ are the wave propagation vectors at $\lambda_{1}$ and $\lambda_{2}$ respectively, $v$ is the speed of light into the medium, $D^{\lambda 2}$ is the diffusion coefficient at the $\lambda_{2}, U_{0}\left(\vec{r}_{s}-\vec{r}, k^{\lambda 1}\right)$ is a term that theoretically describes the established photon field at position $\vec{r}$ into the medium at $\lambda_{1}$ and $G\left(\vec{r}_{d}-\vec{r}, k^{\lambda 2}\right)$ is a term that describes the propagation of the emission photon wave from a fluorochrome at position $\vec{r}$ to the detector. The right-most part of Eq.1 reminds the formulation used in the Rytov approximation for DOT applications and attains similar imaging advantages over a standard Born expansion [7]. The terms $U_{0}, G$, also referred to as the "forward model" are calculated analytically or numerically depending on the complexity of the geometry imaged. Experimentally, we have found that the algorithm of Eq. 1 performs very accurately in phantom measurements [10] and heterogeneous real tissue (c.f. Fig. 2).

\subsection{Fast Forward Model Generation}

While Eq. 1 can be solved analytically for simple boundaries, such as an infinite slab or a cylinder, in-vivo applications require forward models for diffuse media with complex boundaries, typically obtained using numerical methods. Complex geometries are the air-tissue boundaries between diffuse and non-diffuse media and possibly mismatches between diffuse media. While numerical methods offer a convenient method to implement complex boundaries, three-dimensional solutions become impractical due to the large computation times required. Alternative solutions for modeling complex boundaries can be achieved using the tangent-plane method or Kirchoff approximation [11].

The method is simply explained if we assume a diffusive object of volume $\mathrm{V}$ surrounded by a non-diffuse medium. This object is delimited by a surface $\mathrm{S}$, defined by a unit normal $\vec{n}(\vec{r})$ at each point of the interface. The Kirchhoff Approximation (KA) assumes that the surface is replaced at each point by its tangent plane. Therefore, the value of the total average intensity $U$ at any point of the surface $S$ is given by the sum of the homogeneous incident intensity $U_{i n c}$ and the wave reflected from the local plane defined by the surface normal $\vec{n}\left(\vec{r}_{p}\right)$ at that surface point. So as to obtain the expression for the reflected wave, the reflection coefficient for diffusive/non-diffusive interfaces is used [12]. Taking into account the boundary condition at a diffusive/non-diffusive interface [13], $U=-C_{n d} D \partial U / \partial n$, the total intensity measured inside volume $\mathrm{V}$ assuming a source at $\vec{r}_{s}$, and a detector at $\vec{r}_{d}$, is expressed as: 


$$
\begin{aligned}
& U^{K A}\left(\overrightarrow{\mathbf{r}}_{d}\right)=U^{(i n c)}\left(\overrightarrow{\mathbf{r}}_{s}-\overrightarrow{\mathbf{r}}_{d}\right)+ \\
& +\frac{\Delta S}{4 \pi} \sum_{p=1}^{N}\left[C_{n d} D \frac{\partial g\left(\kappa\left|\overrightarrow{\mathbf{r}}_{p}-\overrightarrow{\mathbf{r}}_{d}\right|\right)}{\partial \overrightarrow{\mathbf{n}}_{p}}+g\left(\kappa\left|\overrightarrow{\mathbf{r}}_{p}-\overrightarrow{\mathbf{r}}_{d}\right|\right)\right] \frac{\partial U^{K A}\left(\overrightarrow{\mathbf{r}}_{p}\right)}{\partial \overrightarrow{\mathbf{n}}_{p}} .
\end{aligned}
$$

In Eq. (2), $U^{K A}$ represents the total intensity given by the $\mathrm{KA}, \mathrm{g}$ is the infinite homogeneous Green's function, $\mathrm{D}$ is the diffusion coefficient and $C_{n d}$ is a constant that takes into account the index or refraction mismatch between the diffusive and the non-diffusive medium [13]. The surface values $\partial U^{K A} / \partial \vec{n}$ are given in terms of the local reflection coefficient $R_{n d}$ and the incident intensity by:

$$
\frac{\partial G^{K A}\left(\vec{r}_{s}, \vec{r}_{p}\right)}{\partial \hat{n}_{p}}=\int_{-\infty}^{+\infty}\left[1-R_{N D}(\vec{K})\right] \frac{\partial \tilde{U}^{(i n c)}(\vec{K}, \bar{Z})}{\partial \bar{Z}} \exp (i \vec{K} \cdot \bar{R}) d \vec{K},
$$

where $(\bar{R}, \bar{z})$ are the coordinates of $\left|\vec{r}_{s}-\vec{r}_{p}\right|$ with respect to the plane defined by $\vec{n}\left(\vec{r}_{p}\right)$, namely $\bar{Z}=\left(\vec{r}_{p}-\vec{r}_{p}\right) \cdot\left[-\vec{n}\left(\vec{r}_{p}\right)\right], \bar{R}=\bar{Z}-\left(\vec{r}_{s}-\vec{r}_{p}\right)$. An analogous expression to Eq. (2) can be found for diffusive/diffusive interfaces by means of the diffusive/diffusive reflection and transmission coefficients derived in Ref.[12].

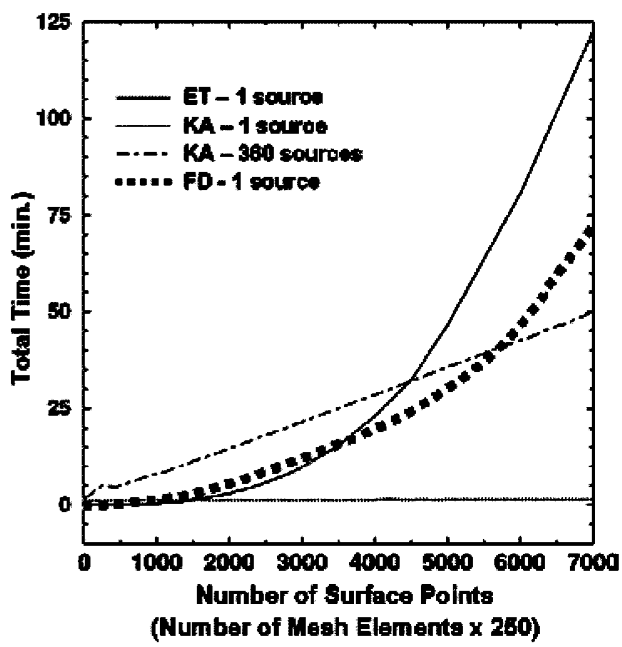

Fig. 1: Computation time of the KA method versus an exact solution using the extinction theorem (ET) and a finite-differences solution (FD).
Figure 1 shows the comparison of the KA method (for 1 and 360 sources) with a) an exact solution using the extinction theorem (ET) [11] and a single source and b) a finite-difference (FD) solution of the diffusion equation for a single source and identical geometrical parameters. The volume simulated was a $2 \mathrm{~cm}$ diffuse cylinder with varying height (surface elements for KA and ET and mesh elements for FD) from 0 to $10 \mathrm{~cm}$. As the number of sources and cylinder height increases, the KA approximation becomes the only viable solution of the three methods for realistic implementations. FD and KA simulations were run to achieve accuracy within $2 \%$ of the exact solution. 


\subsection{Inverse Problem}

For reconstruction purposes equation Eq. 1 was discretized into a number of volume elements (voxels), which yielded a set of coupled linear equations [10]. The terms $U_{0}$ and $G$ were then calculated using Eq.2 for the appropriate boundary conditions and background optical properties at $\lambda_{1}, \lambda_{2}$ respectively. The resulting system of equations was then inverted for the unknown quantity $n(\vec{r})$ using the algebraic reconstruction technique with positive restriction (since no negative fluorescence concentration exists) [7]. There are several other ways to solve the inverse problem such as direct inversion, $\chi^{2}$ - based minimization but we have found that the algebraic reconstruction techniques offer a time-efficient and robust approach.

\section{Imaging Results}

The above algorithms have been recently evaluated with phantom measurements [14]. In addition FMT has been recently used for imaging of cathepsin $B$ activity in nude mice implanted with appropriate tumors known to up-regulate certain proteases [15]. Here we show the results obtained using Eq.1 -Eq.3 in imaging a cylindrical geometry containing an HT1080 tumor implanted in the mammary fat pad of a nude mouse. MR imaging on the same animal was also performed for data validation as well as immunohistochemistry and Western blotting in order to verify the FMT findings. To facilitate optimum photon coupling the animal was immersed in a cylindrical bore that was filled with water, $\mathrm{TiO}_{2}$ particles and India Ink to match the optical properties of the mouse. Figure 2a depicts the correlative axial MR image obtained using a T1 weighted spin echo sequence (TR/TE $300 \mathrm{msec} / 13 \mathrm{msec}$ ). The MR slice shown passes through the tumor region and demonstrates the geometry used. Here a single image is shown although volumetric imaging was performed. Figure $1 \mathrm{~b}$ depicts an axial FMT image from the mouse examined using a cathepsin $\mathrm{B}$ sensitive NIR activatable probe [8]. Fig. 1b depicts an axial FMT image and Fig. 1c depicts a superposition of the MR and FMT images. The bright white spot shown in the left bottom quadrant of the MR image is a fiducial marker containing water and $\mathrm{CuSO} 4$ that was used for registration purposes. The tumor was well resolved by FMT and demonstrated significant cathepsin B over-expression. Western blotting and immunohistochemistry verified the increased cathepsin B concentration in the tumor of investigation (results not shown). The total time for a full three-dimensional reconstruction of 5 FMT planes (slices) was approximately 2 minutes on a Pentium 4 $1.7 \mathrm{Ghz}$ processor. Currently other tumor models with smaller tumors and deeper implantation are investigated. 

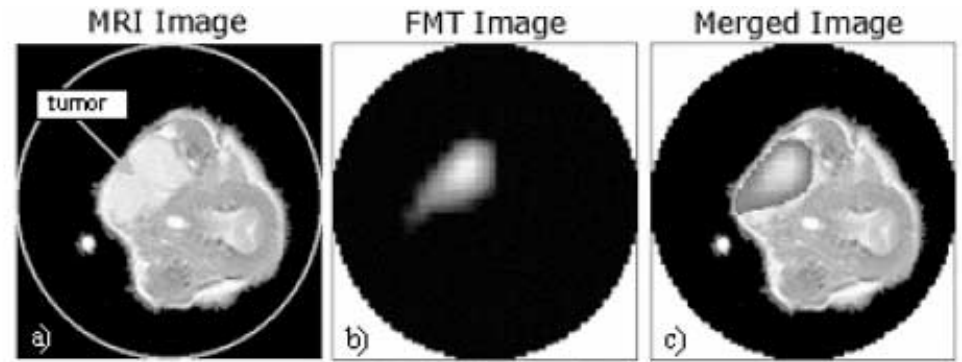

$\begin{array}{llllllll}0 & 0.2 & 0.4 & 0.6 & 0.8 & 1.0 & 1.2\end{array}$

Fig. 2. In-vivo tomography of cathepsin B expression. a) MRI image passing through an HT1080 tumor implanted in the mammary fat pad of a nude mouse. b) Corresponding FMT image c) Superposition of a) and b).

\section{Discussion and Conclusion}

FMT offers a platform for interrogating targeted molecular events in tissue and offers unique features that are very attractive for animal research and clinical imaging. First, it can penetrate for several centimetres into tissue, therefore it is not limited to imaging surface events. It has been predicted recently that NIRF signals could propagate for more than 15 centimetres in breast tissue and lung and more than $5 \mathrm{~cm}$ in the adult brain [16]. Therefore the technique could be applied to imaging large organs. Second, it allows the quantification of NIRF probes, which translates into quantitative insights of enzymatic activity. Third, it offers excellent molecular specificity and target to background ratio by capitalizing on fluorescence dequenching of the employed NIRF probes. Fourth, it offers very sensitive photon detection (fempto-mole quantities for millimetre sized objects). Finally, it can be engineered to simultaneously target multiple specific molecular processes by imaging appropriate NIRF probes at different wavelengths and uses optical technology, which is inexpensive, easy to built, safe and does not use ionising radiation.

In this paper we demonstrated that time-efficient reconstructions can be achieved in three-dimensional problems and that the combination of Eq.1 - Eq.3 can yield accurate reconstruction results in in-vivo imaging of molecular function. A technique capable of imaging and quantifying enzymatic activity in deep tissues should prove to be a powerful diagnostic and treatment guidance tool in clinical medicine. It is expected that the propagation of fluorescent probes into clinical examinations, will be directly associated with the development of clinical FMT systems for diagnosis and treatment planning. The use of safe radiation and the cost-efficiency of the development also meet successfully the practical aspects of a clinical implementation. Therefore FMT could propagate quickly in research and perhaps the everyday clinical interrogation of basic functional and molecular processes. 


\section{Acknowledgements}

V.N gratefully acknowledges support in-part from fellowship DRG-1638 of the Cancer Research Fund of the Damon Runyon-Walter Winchell Foundation and the US Army CDMRP BC995360 concept award. R.W. acknowledges support from the NIH P50 CA86355 grant.

\section{References}

1. Weissleder, R., A clearer vision for in vivo imaging. Nature Biotechnology, 2001. 19(4): p. 316-317.

2. Budinger, T.F., D.A. Benaron, and A.P. Koretsky, Imaging transgenic animals. Annual Review of Biomedical Engineering, 1999. 1: p. 611-648.

3. Korlach, J., et al., Characterization of lipid bilayer phases by confocal microscopy and fluorescence correlation spectroscopy. Proc Natl Acad Sci, 1999. 96(15): p. 8461-6.

4. Rajadhyaksha, M., et al., In vivo confocal scanning laser microscopy of human skin: melanin provides strong contrast. J Invest Dermatol, 1995. 104(6): p. 946-52.

5. Toomre, D. and D.J. Manstein, Lighting up the cell surface with evanescent wave microscopy. Trends in Cell Biology, 2001. 11(7): p. 298-303.

6. Arridge, S.R., Optical tomography in medical imaging. Inverse Problems, 1999. 15(2): p. R41-R93.

7. Kak, A. and M. Slaney, Principles of Computerized tomographic imaging. 1988, New York: IEEE Press.

8. Weissleder, R., et al., In vivo imaging of tumors with protease-activated near-infrared fluorescent probes. Nature Biotech, 1999. 17(4): p. 375-8.

9. Tyagi, S., S.A.E. Marras, and F.R. Kramer, Wavelength-shifting molecular beacons. Nature Biotechnology, 2000. 18(11): p. 1191-1196.

10.Ntziachristos, V. and R. Weissleder, Experimental three-dimensional fluorescence reconstruction of diffuse media using a normalized Born approximation. Optics Letters, 2001. 26(12): p. 893-895.

11.Ripoll, J., et al., Kirchhoff approximation for diffusive waves. Phys Rev E, 2001. 64(5 Pt 1): p. 051917.

12.Ripoll, J. and M. Nieto-Vesperinas, Reflection and transmission coefficients for diffuse photon density waves. Optics Letters, 1999. 24: p. 796-798.

13.Aronson, R., Boundary conditions for diffusion of light. J Opt Soc Am A, 1995. 12(11): p. 2532-9.

14.Ripoll, J., et al., A fast analytical method for optical tomography in diffusive media with arbitrary geometry. Optics Letters, 2002. 27(7), 527-529.

15.Ntziachristos, V., et al., Fluorescence-mediated tomography resolves protease activity in vivo. Nature Medicine, July 2002. in press.

16.Ntziachristos, V., J. Ripoll, and R. Weissleder, Would near-infrared fluorescence signals propagate through large human organs for clinical studies. Optics Letters, 2002. 27(5), 333 335. 